\title{
Cholesterol-Years Score is Associated with Development of Senile Degenerative Aortic Stenosis in Heterozygous Familial Hypercholesterolemia
}

\author{
Tsuyoshi Nozue ${ }^{1}$, Masa-aki Kawashiri ${ }^{1}$, Toshinori Higashikata ${ }^{1}$, Atsushi Nohara $^{2}$, Akihiro Inazu $^{3}$, \\ Junji Kobayashi ${ }^{4}$, Junji Koizumi ${ }^{5}$, Masakazu Yamagishi ${ }^{1}$, and Hiroshi Mabuchi ${ }^{2}$
}

\footnotetext{
${ }^{1}$ Molecular Genetics of Cardiovascular Disorders, Division of Cardiovascular Medicine, Graduate School of Medical Science, Kanazawa University, Kanazawa, Japan.

${ }^{2}$ Department of Lipidology, Graduate School of Medical Science, Kanazawa University, Kanazawa, Japan.

${ }^{3}$ Department of Health Science, Kanazawa University, Kanazawa, Japan.

${ }^{4}$ Department of Lifestyle-related Disease, Graduate School of Medical Science, Kanazawa University, Kanazawa, Japan.

${ }^{5}$ Department of General Medicine, Kanazawa University Hospital, Kanazawa, Japan.
}

\begin{abstract}
We retrospectively evaluated the frequency and identified the factors associated with the development of aortic stenosis (AS) in 96 patients with heterozygous familial hypercholesterolemia (FH). The frequency of AS was $31 \%(4 / 13)$ and that of critical stenosis was $15 \%(2 / 13)$ in older patients over the age of 70 years. All 4 patients with AS were female aged more than 70 years who were diagnosed with $\mathrm{FH}$ when aged more than 60 years. There were no significant differences in conventional coronary risk factors; however, the age at cardiac catheterization, age at diagnosis of $\mathrm{FH}$ and the cholesterol-years score (CYS) with AS were significantly higher than those without AS ( $p=0.006, p=0.017$, $p=0.021$, respectively). In multiple regression analysis, CYS was a significant independent predictor for the development of AS $(p=0.037)$ in 13 older patients over the age of 70 years. These results suggest that physicians should be aware that AS needs attention in older patients with heterozygous $\mathrm{FH}$, especially women who have been diagnosed late in life and those who have been inadequately treated.
\end{abstract}

J Atheroscler Thromb, 2006; 13:323-328.

Key words; Familial hypercholesterolemia, Aortic stenosis, Cholesterol-years score, Statins

\section{Introduction}

Before 1950, rheumatic disease was considered to be the predominant cause of valvular aortic stenosis (AS). Acute rheumatic fever had virtually vanished by 1950, thus the number of patients with chronic rheumatic valvular AS was correspondingly low. Currently, two major causes of AS are considered, bicuspid aortic valve and degenerative calcific changes of tricuspid

Address for correspondence: Tsuyoshi Nozue, Molecular Genetics of Cardiovascular Disorders, Division of Cardiovascular Medicine, Graduate School of Medical Science, Kanazawa University 13-1 Takara-machi, Kanazawa 921-8241, Japan.

E-mail: nozue2493@yahoo.co.jp

Received: July 5, 2006

Accepted for publication: October 5, 2006 aortic valve ${ }^{1)}$. Most of the risk factors of valvular AS are common to those of atherosclerotic cardiovascular disorders, such as hypercholesterolemia, hypertension, male sex and older persons ${ }^{2}$. Meta-analysis of 33 studies showed that $37 \%$ of coronary artery disease (CAD) was complicated with $\mathrm{AS}^{3)}$. Conversely, patients with AS who needed aortic valve replacement surgery much more frequently possessed CAD that required bypass surgery ${ }^{1)}$. The histological features of AS include calcification, fibrosis, and lipid deposition with morphological similarities to atherosclerosis ${ }^{4)}$. This suggests that atherogenic factors may play an important role in the development of AS.

Familial hypercholesterolemia $(\mathrm{FH})$ is a common autosomal dominant disorder caused by a mutation of the gene for the low-density lipoprotein (LDL) receptor $^{5)}$. FH is frequently associated with premature CAD, 
and the rate of death from CAD among heterozygotes is several times higher than that among the general population ${ }^{5-8)}$. It has been proved that the increased use of lipid-lowering drugs, especially 3-hydroxy-3-methylglutaryl coenzyme A (HMG-CoA) reductase inhibitors (statins), has improved the prognosis of $\mathrm{CAD}^{9)}$; however, a recent double-blind, placebo-controlled study using $80 \mathrm{mg}$ of atorvastatin showed no advantage of aggressive lipid-lowering therapy to halt the progression of $\mathrm{AS}^{10)}$.

Valvular and supra-valvular AS has been observed frequently in homozygous $\mathrm{FH}^{11,12)}$; however, little is known about the frequency and the factors associated with the development of AS in heterozygous FH. In the present study, we retrospectively evaluated the frequency and identified the factors associated with the development of AS in patients with heterozygous FH.

\section{Subjects and Methods}

\section{Subjects}

We retrospectively studied 96 consecutive patients with heterozygous FH (male/female $=66 / 30$, mean age $=56 \pm 13$ years) who received elective cardiac catheterization between January 1990 and December 2000.

$\mathrm{FH}$ was diagnosed according to the following two criteria: (1) primary hypercholesterolemic patients (arbitrarily above $230 \mathrm{mg} / \mathrm{dL}$ in any age group) with tendon xanthomas, or (2) primary hypercholesterolemic patients with and without tendon xanthomas in a first-degree relative of familial hypercholesterolemic patients ${ }^{13}$. All the data of these patients were obtained before introducing lipid-lowering therapy.

AS was diagnosed according to the following two criteria: (1) presence of calcification and thickening of the aortic valve by B-Mode echocardiography, and (2) peak aortic jet velocity $>2 \mathrm{~m} / \mathrm{s}$ by continuous wave Doppler echocardiography. No patients had bicuspid aortic valve or a past history of rheumatic fever.

\section{Laboratory Determination}

Blood samples were obtained after overnight fasting. Serum cholesterol, triglyceride, and high-density lipoprotein (HDL) cholesterol levels were determined by standard enzymatic methods. LDL cholesterol was calculated by Friedewald's formula ${ }^{14}$. Lp(a) was assayed by commercially available enzyme-linked immunosorbent assay (Tint Elize, Biopool, Sweden).

To estimate the lifelong total vascular exposure to profound hypercholesterolemia, the cholesterol-years score (CYS) was calculated ${ }^{15)}$ as follows. The total cholesterol concentration $(\mathrm{mg} / \mathrm{dL})$ of each patient at the time of the original diagnosis was multiplied by
Table 1. Baseline characteristics

\begin{tabular}{lc} 
Age (years) & $56 \pm 13$ \\
Sex $($ male/female) & $66 / 30$ \\
HT $(\mathrm{cm})$ & $159.1 \pm 10.1$ \\
BW $(\mathrm{kg})$ & $61.1 \pm 10.5$ \\
BMI $\left(\mathrm{kg} / \mathrm{m}^{2}\right)$ & $24.0 \pm 2.8$ \\
Achilles tendon thickness & \\
$\quad$ rt $(\mathrm{mm})$ & $13 \pm 5$ \\
lt $(\mathrm{mm})$ & $14 \pm 5$ \\
At original diagnosis & \\
TC $(\mathrm{mg} / \mathrm{dL})$ & $330 \pm 69$ \\
$\quad$ LDL-C (mg/dL) & $256 \pm 65$ \\
At cardiac catheterization & \\
TC $(\mathrm{mg} / \mathrm{dL})$ & $259 \pm 53$ \\
LDL-C (mg/dL) & $200 \pm 52$ \\
\hline
\end{tabular}

HT, height; BW, body weight; BMI, body mass index; TC, total cholesterol; LDL-C, low-density lipoprotein cholesterol

the age of the patient at diagnosis. The total cholesterol concentration $(\mathrm{mg} / \mathrm{dL})$ of each patient after therapy was then multiplied by the years of treatment. The pretreatment and post-treatment cholesterol-years (mg-y/ $\mathrm{dL}$ ) were then added together for the total CYS.

\section{Echocardiography}

All patients were studied with B-Mode, M-Mode, Doppler (pulsed and continuous Mode) and color Doppler echocardiography using a Toshiba SSA-380A electronic scanner or Toshiba SSH-160A scanner with a 3.75 or $2.5 \mathrm{MHz}$ transducer. We obtained an almost complete range of echocardiographic studies. Calcification and thickening of the aortic valve were assessed in short- and long-axis two-dimensional views.

\section{Cardiac Catheterization}

Coronary angiography was performed with multiple projection. Coronary angiograms were interpreted by at least 2 cardiologists without knowledge of the patient's clinical and laboratory findings. CAD was defined as the presence of a $50 \%$ or greater diameter reduction of at least 1 coronary artery. The extent and severity of stenotic changes were assessed by a score assigned to each of 15 segments according to the classification of the American Heart Association Grading Committee. A normal coronary angiogram was graded as 0 , stenosis of less than $25 \%$ as $1,25 \%$ to $50 \%$ stenosis as $2,50 \%$ to $75 \%$ stenosis as 3 , and more than $75 \%$ stenosis as 4 . The coronary stenosis index (CSI) was defined as the sum of these scores, with a maximal value of $60^{16)}$.

Left ventriculography was performed and the 
Table 2. Clinical characteristics of patients with aortic stenosis

\begin{tabular}{|c|c|c|c|c|c|c|c|}
\hline Case & Age (years) & Sex & \multicolumn{2}{|c|}{ Achilles tendon thickness } & $\begin{array}{c}\text { Age at diagnosis } \\
\text { of FH (years) }\end{array}$ & \multicolumn{2}{|c|}{ At original diagnosis } \\
\hline 2 & 75 & Female & 20 & 19 & 66 & 405 & 316 \\
\hline 3 & 75 & Female & 12 & 12 & 71 & 283 & 230 \\
\hline 4 & 71 & Female & 11 & 11 & 61 & 343 & 260 \\
\hline
\end{tabular}

TC, total cholesterol; LDL-C, low-density lipoprotein cholesterol

pressure gradient across the aortic valve was measured in all patients. Thoratic aortography was performed in cases with AS by echocardiography.

\section{Statistical Analysis}

Statistical analysis was performed using Statview 5.0 software (SAS Institute Inc., Cary, North Carolina). All values are expressed as the mean \pm SD. Differences between the means were compared with the unpaired $t$-test. The significance of any differences in proportions was tested with Chi-squared analysis. For stepwise multiple regression analysis, the nominal variable (hypertension, glucose intolerance, sex, and the presence of CAD) was included as well as numerical variables (total cholesterol levels at original diagnosis and at cardiac catheterization, body mass index, age at diagnosis of FH, CSI, and CYS). A statistically significant difference was defined as $p<0.05$.

\section{Results}

Table 1 shows the baseline characteristics in this study. Sixty-six patients were male, and another $30 \mathrm{pa}-$ tients were female with a mean age of $56 \pm 13$ years. Mean total and LDL cholesterol levels at original diagnosis were $330 \pm 69 \mathrm{mg} / \mathrm{dL}$ and $256 \pm 65 \mathrm{mg} / \mathrm{dL}$. Eight patients had been treated with only diet, 52 patients with statins alone, 12 patients with probucol alone, 9 patients with both statins and resins, 15 patients with both statins and probucol. Mean total and LDL cholesterol levels at cardiac catheterization significantly decreased to $259 \pm 53 \mathrm{mg} / \mathrm{dL}$ and $200 \pm 52 \mathrm{mg} /$ $\mathrm{dL}$, respectively.

Four patients were diagnosed with AS. Table 2 shows the clinical characteristics of 4 patients with AS. All were female aged more than 70 years who were diagnosed with $\mathrm{FH}$ when aged more than 60 years. Table 3 shows the summary of cardiac-catheterization findings of patients with AS. None had supra-valvular AS, nor ostial coronary stenosis. Two patients showed critical stenosis $>50 \mathrm{mmHg}$ of pressure gradient.
Table 3. Summary of cardiac-catheterization findings of patients with aortic stenosis

\begin{tabular}{|c|c|c|c|c|c|}
\hline \multirow[b]{2}{*}{ Case } & \multirow{2}{*}{ NDV } & \multirow{2}{*}{ CSI } & \multicolumn{2}{|c|}{ AS } & \multirow{2}{*}{$\begin{array}{l}\text { Peak-to-peak pressure } \\
\text { gradient }(\mathrm{mmHg})\end{array}$} \\
\hline & & & $\begin{array}{l}\text { Supra- } \\
\text { valvular }\end{array}$ & Valvular & \\
\hline 1 & 0 & 9 & No & Yes & 84 \\
\hline 2 & 3 & 34 & No & Yes & 63 \\
\hline 3 & 2 & 25 & No & Yes & 18 \\
\hline 4 & 2 & 20 & No & Yes & 17 \\
\hline
\end{tabular}

NVD, number of diseased vessels; CSI, coronary stenosis index

As all 4 patients with AS were female, we compared the clinical characteristics of 30 women in order to evaluate the factors associated with the development of AS (Table 4). There were no significant differences in conventional coronary risk factors such as total and LDL cholesterol levels, fasting plasma glucose, immunoreactive insulin, $\mathrm{Lp}(\mathrm{a})$, homocysteine, or systolic and diastolic blood pressure. However, the age at cardiac catheterization, age at diagnosis of $\mathrm{FH}$ and the CYS with AS were significantly higher than in those without AS $(p=0.006, p=0.017, p=0.021$, respectively).

To balance the contribution from aging, multiple regression analysis was performed using 10 variables: total cholesterol levels at original diagnosis and at cardiac catheterization, hypertension, glucose intolerance, body mass index, sex, age at diagnosis of $\mathrm{FH}$, presence of CAD, CSI, and CYS in 13 elder patients over the age of 70 years. In this analysis, CYS was a significant $(p=0.037)$ independent predictor for the development of AS (Table 5). When we performed multiple regression analysis except for this CYS, female sex was an independent but not significant $(p=0.0642)$ factor for the development of AS (data not shown).

\section{Discussion}

In our study, the frequency of AS was 31\% (4/13) 
Table 4. Clinical characteristics of patients with and without aortic stenosis in 30 women

\begin{tabular}{lccc}
\hline \multicolumn{1}{c}{ Variable } & AS $(+)(\mathrm{n}=4)$ & AS $(-)(\mathrm{n}=26)$ & $p$ Value \\
\hline Age (years) & $75 \pm 3$ & $61 \pm 9$ & 0.006 \\
Age at diagnosis of FH (years) & $66 \pm 4$ & $51 \pm 12$ & 0.017 \\
Onset of CAD (years) & $71 \pm 5$ & $62 \pm 6$ & 0.047 \\
At original diagnosis & & & \\
$\quad$ TC (mg/dL) & $351 \pm 52$ & $355 \pm 79$ & 0.936 \\
$\quad$ LDL-C (mg/dL) & $278 \pm 41$ & $275 \pm 84$ & 0.933 \\
At cardiac catheterization & & & \\
$\quad$ TC (mg/dL) & $232 \pm 17$ & $267 \pm 53$ & 0.204 \\
$\quad 174 \pm 14$ & $207 \pm 54$ & 0.239 \\
FPG (mg/dL) $(\mathrm{mg} / \mathrm{dL})$ & $95 \pm 21$ & $105 \pm 41$ & 0.624 \\
IRI (IU/L) & $5.2 \pm 1.0$ & $7.9 \pm 3.2$ & 0.119 \\
Lp (a) (mg/dL) & $15.5 \pm 13.2$ & $25.3 \pm 15.3$ & 0.347 \\
Hcy (mg/dL) & $9.8 \pm 2.1$ & $10.0 \pm 7.4$ & 0.969 \\
SBP (mmHg) & $133 \pm 23$ & $123 \pm 18$ & 0.338 \\
DBP (mmHg) & $70 \pm 13$ & $70 \pm 10$ & 0.961 \\
CSI & $22 \pm 10$ & $15 \pm 12$ & 0.291 \\
CYS (mg-y/dL) & $25115 \pm 3538$ & $20238 \pm 3746$ & 0.021 \\
\hline
\end{tabular}

CAD, coronary artery disease; TC, total cholesterol; LDL-C, low-density lipoprotein cholesterol; FPG, fasting plasma glucose; IRI, immuno reactive insulin; Hcy, homocysteine; SBP, systolic blood pressure; DBP, diastolic blood pressure; CSI, coronary stenosis index; CYS, cholesterol-years score

Table 5. Stepwise multiple regression coefficients, F values, and adjusted $\mathrm{R}^{2}$ for the development of aortic stenosis in 13 older patients

\begin{tabular}{cccccc}
\hline Step Variable & Coefficient & SE & F Value & Adjusted $\mathrm{R}^{2}$ & $p$ Value \\
\hline 1 CYS & 7.9 & 3.3 & 5.7 & 0.28 & 0.037 \\
\hline
\end{tabular}

Multiple regression analysis was performed using 10 variables: total cholesterol level at original diagnosis and at cardiac catheterization, hypertension, glucose intolerance, body mass index, sex, age at diagnosis of $\mathrm{FH}$, presence of coronary artery disease, coronary stenosis index, and cholesterol-years score. Hypertension, glucose intolerance, male and presence of coronary artery disease were assigned values of 1 . Normotension, normal glucose intolerance, female and no presence of coronary artery disease were assigned values of 0 .

and that of critical stenosis was 15\% (2/13) in older patients over the age of 70 years. It is known that age contributes to degenerative thickening of the aortic valve in the general population ${ }^{17)}$ and calcific deposits in the aortic valve are common in elderly persons and may lead to valvular $\mathrm{AS}^{18-20)}$. Lindroos et al. ${ }^{17)}$ reported that the prevalence of at least moderate valvular AS was about $5 \%$ and that of critical stenosis was about $3 \%$ in 501 of the general population aged 75 to 86 years. Aronow et al. ${ }^{21)}$ reported that the prevalence of severe valvular AS was $2 \%$ in 781 unselected elderly patients in a health care facility. Compared with these data, the prevalence of AS in older patients with het- erozygous FH was higher than that of previous studies suggesting that hypercholesterolemia might be an increased risk factor for the development of AS.

Our data showed that CYS was a significant independent predictor for the development of AS. Rallidis et al. ${ }^{22)}$ showed a significant positive correlation between the mean gradient across the aortic valve and total CYS in heterozygous FH. This correlation indicates that lifelong exposure to high cholesterol concentrations accelerated age-related degeneration of the aortic valve; therefore, it would be necessary to start lipid-lowering therapy early in life and to control cholesterol level adequately.

The mean age of death in heterozygous $\mathrm{FH}$ men and women was 54 and 65 years, respectively ${ }^{8,23,24)}$. Many clinical trials have shown that statins can significantly reduce the incidence of CAD in both primary and secondary prevention ${ }^{25-28)}$; thus, statins revolutionized the prognosis of CAD in hypercholesterolemic patients. However, mortality from CAD in statintreated patients with $\mathrm{FH}$ is 2.5 to 2.6-fold higher than in the general population ${ }^{29,30)}$. This mortality risk is highest in middle-aged men. On the other hand, female $\mathrm{FH}$ patients have no increased mortality from CAD compared to the general population ${ }^{29)}$. Interestingly, this sex difference, which is also a characteristic feature of the usual form of CAD in the general population, does not seem to be operative in homozygous 
FH. Estrogens have been reported to provide protection against $\mathrm{CAD}$ in postmenopausal women ${ }^{31)}$. In consideration of our data, there is some possibility that the anti-atherogenic effects of estrogens are attributed to senile degenerative aortic valve stenosis in these postmenopausal female patients.

In previous reports, statins had reduced progression of $\mathrm{AS}^{32)}$ and were associated with slower progression of $\mathrm{AS}^{32)}$; however, there was no association between the progression of AS and the cholesterol lev$\mathrm{el}^{33)}$. A recent randomized trial of intensive lipid-lowering therapy did not halt the progression of AS ${ }^{10)}$. AS is common in younger patients with homozygous $\mathrm{FH}$ and it appears that they are exposed to high cholesterol concentrations early in life. Conversely, AS occurs only in cases of severe and prolonged hypercholesterolemia in heterozygous $\mathrm{FH}^{22}$. Thus, both the cholesterol level and duration affect the development of AS in heterozygous $\mathrm{FH}$.

\section{Conclusions}

In conclusion, CYS was a significant independent predictor for the development of senile degenerative AS in heterozygous FH. Physicians should be aware that AS needs attention in older patients with heterozygous $\mathrm{FH}$, especially in women who have been diagnosed late in life and those who have been inadequately treated. Further studies are necessary to discover the mechanisms and factors for the prediction of senile degenerative AS.

\section{Study Limitations}

There are some limitations regarding the interpretation of these results and drawing conclusions from a case series. First, a relatively small number of patients eligible for analysis in this cohort study makes it difficult to generalize or to apply the results to other patient populations. Second, all patients in this study were examined by a cardiologist, who decided that elective cardiac catheterization was indicated. Third, there will be a survivor bias since the most severely affected patients may have died at home, and were inevitably excluded.

\section{Acknowledgements}

We express our special thanks to Mayumi Yoshida, Mihoko Mizuno, and Sachio Yamamoto for their outstanding technical assistance. We are grateful to Dr. Ichiro Michishita (Division of Cardiology, Department of Internal Medicine, Yokohama Sakae Kyosai Hospital) for advice in preparing this manuscript.

\section{References}

1) Davies MJ, Treasure T, and Parker DJ: Demographic characteristics of patients undergoing aortic valve replacement for stenosis: relation to valve morphology. Heart, 1996; 75:174-178

2) Otto CM, Lind BK, Kitzman DW, Gersh BJ, and Siscovick DS: Association of aortic-valve sclerosis with cardiovascular mortality and morbidity in the elderly. $\mathrm{N}$ Engl J Med, 1999; 341:142-147

3) Mautner GC and Roberts WC: Reported frequency of coronary arterial narrowing by angiogram in patients with valvular aortic stenosis. Am J Cardiol, 1992; 70:539-540

4) Otto CM, Kuusisto J, Reichenbach DD, Gown AM, and O'Brien KD: Characterization of the early lesions of "degenerative" valvular aortic stenosis. Histological and immunohistochemical studies. Circulation, 1994; 90:844-853

5) Goldstein JL, Hobbs HH, and Brown MS: Familial hypercholesterolemia. In: The Metabolic Basis of Inherited Disease. 8th Ed, ed by Scriver CR, Beaudet AL, Sly WS, Valle I, pp 2863-2913, McGraw-Hill, New York, 2001

6) Jensen J, Blankenhorn DH, and Kornerup V: Coronary disease in familial hypercholesterolemia. Circulation, 1967; 36:77-82

7) Stone NJ, Levy RI, Fredrickson DS, and Verter J: Coronary artery disease in 116 kindred with familial type II hyperlipoproteinemia. Circulation, 1974; 49:476-488

8) Mabuchi H, Miyamoto S, Ueda K, Oota M, Takegoshi T, Wakasugi $T$, and Takeda R: Causes of death in patients with familial hypercholesterolemia. Atherosclerosis, 1986; 61:1-6

9) Scientific Steering Committee on behalf of the Simon Broome Register Group: Mortality in treated heterozygous familial hypercholesterolaemia; implications for clinical management. Atherosclerosis, 1999; 142:105-112

10) Cowell SJ, Newby DE, Prescott RJ, Bloomfield P, Reid J, Northridge DB, and Boon NA; Scottish Aortic Stenosis and Lipid Lowering trial, Impact on Regression (SALTIRE) Investigators: A randomized trial of intensive lipid-lowering therapy in calcific aortic stenosis. N Engl J Med, 2005; 352:2389-2397

11) Haitas B, Baker SG, Meyer TE, Joffe BI, and Seftel HC: Natural history and cardiac manifestations of homozygous familial hypercholesterolemia. Q J Med, 1990; 76:731-740

12) Allen JM, Thompson GR, Myant NB, Steiner R, and Oakley CM: Cardiovascular complication of homozygous familial hypercholesterolemia. Br Heart J, 1980; 44:361-368

13) Mabuchi H, Ito $S$, Haba T, Ueda K, and Ueda R: Discrimination of familial hypercholesterolemia and secondary hypercholesterolemia by Achilles' tendon thickness. Atherosclerosis, 1977; 28:61-68

14) Friedewald WT, Levy RI, and Fredrickson DS: Estimation of the concentration of low-density lipoprotein cholesterol in plasma, without use of the preparative ultracentrifuge. Clin Chem, 1972; 18:499-502

15) Hoeg JM, Feuerstein IM, and Tucker EE: Detection and quantitation of calcific atherosclerosis by ultrafast computed tomography in children and young adults with homozygous familial hypercholasterolemia. Arterioscler Thromb, 1994; 14:1066-1074 
16) Tatami R, Mabuchi H, Ueda K, Ueda R, Haba T, Kametani T, Ito S, Koizumi J, Ohta M, Miyamoto S, Nakayama A, Kanaya H, Oiwake H, Genda A, and Takeda R: Intermediate-density lipoprotein and cholesterol-rich very low density lipoprotein in angiographically determined coronary artery disease. Circulation, 1981; 64:1174-1184

17) Lindroos M, Kupari M, Heikkila J, and Tilvis R: Prevalence of aortic valve abnormalities in the eldely: an echocardiographic study of a random population sample. J Am Coll Cardiol, 1993; 21:1220-1225

18) Roberts WC, Perloff JK, and Costantino T: Severe valvular aortic stenosis in patients over 65 years of age. Am J Cardiol, 1971; 27:497-506

19) Waller BF and Roberts WC: Cardiovascular disease in the very elderly. Analysis of 40 necropsy patients aged 90 years or over. Am J Cardiol, 1983; 51:403-421

20) Aronow WS, Schwartz KS, and Koenigsberg M: Correlation of aortic cuspal and aortic root disease with aortic systolic ejection murmurs and with mitral anular calcium in persons older than 62 years in a long-term health care facility. Am J Cardiol, 1986; 58:651-652

21) Aronow WS and Kronzon I: Prevalence and severity of valvular aortic stenosis determined by Dopler echocardiography and its association with echocardiographic and electrocardiographic left ventricular hypertrophy and phisical signs of aortic stenosis in elderly patients. Am J Cardiol, 1991; 67:776-777

22) Rallidis L, Naoumova RP, Thompson GR, and Nihoyannopoulos P: Extent and severity of atherosclerotic involvement of the aortic valve and root in familial hypercholesterolemia. Heart, 1998; 80:583-590

23) Heiberg A: The risk of atherosclerotic vascular disease in subjects with xanthomatosis. Acta Med Scand, 1975; 198:249-261

24) Beaumont V, Jacotot B, and Beaumont JL: Ischemic disease in men and women with familial hypercholesterolemia and xanthomatosis. A comparative study of genetic and environmental factors in 274 heterozygous cases. Atherosclerosis, 1976; 24:441-450

25) Scandinavian Simvastatin Survival Study Group: Randomized trial of cholesterol lowering in 4444 patients with coronary heart disease: the Scandinavian Simvastatin Survival Study (4S). Lancet, 1994; 344:1383-1389

26) Shepherd J, Cobbe SM, Ford I, Isles CG, Lorimer AR, MacFarlane PW, McKillop JH, and Packard CJ: Prevention of coronary heart disease with pravastatin in men with hypercholesterolemia. N Engl J Med, 1995; 333:1301-1307

27) Byington RP, Jukema JW, Salonen JT, Pitt B, Bruschke AV, Hoen H, Furberg CD, and Mancini GB: Reduction in cardiovascular events during pravastatin therapy. Pooled analysis of clinical events of the Pravastatin Atherosclerosis Intervention Program. Circulation, 1995; 92:2419-2425

28) Sacks FM, Pfeffer MA, Moye LA, Rouleau JL, Rutherford JD, Cole TG, Brown L, Warnica JW, Arnold JM, Wun CC, Davis BR, and Braunwald E: The effect of pravastatin on coronary events after myocardial infarction in patients with average cholesterol levels. Cholesterol and Recurrent Events Trial investigators. N Engl J Med, 1996; 335:1001-1009

29) Mohrschladt MF, Westendorp RG, Gevers Leuven JA, and Smelt AH: Cardiovascular disease and mortality in statin-treated patients with familial hypercholesterolemia. Atherosclerosis, 2004; 172:329-335

30) Neil HA, Hawkins MM, Durrington PN, Betteridge DJ, Capps NE, and Humphries SE: Simon Broome Familial Hyperlipidaemia Register Group and Scientific Steering Committee: Non-coronary heart disease mortality and risk of fatal cancer in patients with treated heterozygous familial hypercholesterolemia: a prospective registry study. Atherosclerosis, 2005; 179:293-297

31) Barrett-Connor E: Sex differences in coronary heart disease. Why are woman so superior? The 1995 Ancel Keys Lecture. Circulation, 1997; 95:252-264

32) Novaro GM, Tiong IY, Pearce GL, Lauer MS, Sprecher DL, and Griffin BP: Effect of Hydroxymethylglutaryl coenzyme A reductase inhibitors on the progression of calcific aortic stenosis. Circulation, 2001; 104:2205-2209

33) Bellamy MF, Pellikka PA, Klarich KW, Tajik AJ, and Enriquez-Sarano M: Association of cholesterol levels, hydroxymethylglutaryl coenzyme A reductase inhibitor treatment, and progression of aortic stenosis in the community. J Am Coll Cardiol, 2002; 40:1723-1730 